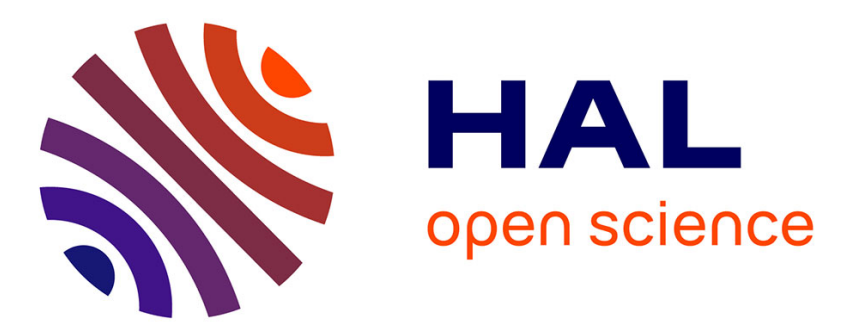

\title{
Distorsion et détournement des dispositifs inclusifs : des obstacles à la transition vers de nouvelles pratiques?
}

Hervé Benoit

\section{To cite this version:}

Hervé Benoit. Distorsion et détournement des dispositifs inclusifs : des obstacles à la transition vers de nouvelles pratiques?. La nouvelle revue de l'adaptation et de la scolarisation, 2013, 61, pp. 49-63. 10.3917/nras.061.0049 . hal-01674673

\section{HAL Id: hal-01674673 \\ https://hal-inshea.archives-ouvertes.fr/hal-01674673}

Submitted on 3 Jan 2018

HAL is a multi-disciplinary open access archive for the deposit and dissemination of scientific research documents, whether they are published or not. The documents may come from teaching and research institutions in France or abroad, or from public or private research centers.
L'archive ouverte pluridisciplinaire HAL, est destinée au dépôt et à la diffusion de documents scientifiques de niveau recherche, publiés ou non, émanant des établissements d'enseignement et de recherche français ou étrangers, des laboratoires publics ou privés. 


\title{
Distorsion et détournement des dispositifs inclusifs: des obstacles à la transition vers de nouvelles pratiques?
}

\author{
Hervé BENOIT \\ INS HEA \\ Laboratoire CRISES (EA 4424) - Esthétique et éthique en psychanalyse \\ Université de Montpellier 3
}

Résumé: L'objet de cet article est de montrer en quoi la dynamique institutionnelle de l'accessibilité du système éducatif, fondée sur un corpus de textes prescriptifs et explicatifs (loi, décrets et circulaires) qui se donnent explicitement pour but de bâtir L'école pour tous, pourrait se trouver engagée dans certaines formes d'impasses du pensé et de l'agi pédagogiques. L'hétérogénéité et l'ambiguïté des textes régissant les dispositifs inclusifs, marqués par des ruptures de cohérence discursive, se combinent et s'articulent dans un discours apocryphe, sous-jacent à l'élaboration par les acteurs (enseignants et personnels d'encadrement) des éléments d'épistémologie pratique. Ce discours professionnel de type subliminal et hybride, dont la figure du déni est l'un des générateurs, est susceptible de renforcer les pratiques intégratives, de faire obstacle à leur transformation et d'entraver la mise en place de formes pédagogiques innovantes en détournant les dispositifs inclusifs de leur finalité. II admet en effet en son sein la coexistence d'éléments ou d'objets contradictoires, tout en s'abstenant de prendre en charge ces contradictions, de sorte que tout se passe comme si elles n'existaient pas.

Mots-clés: Accessibilité pédagogique - Déni - Discordances - Discours - Dispositif - Distorsion - Epistémologie pratique - Hybridation - Inclusion - Innovation - Intégration - Intertextualité - Résistance.

Distortion and misuse of inclusive methods: are they obstacles to transition from integration to new practices?

Summary: The purpose of this article is to show how the institutional dynamic of accessibility in the educational system, based on a corpus of prescriptive and explanatory texts (laws, decrees and circulars) which explicitly seek to build "education for all" might find itself in certain theoretical and practical impasses. The heterogeneity and ambiguity of the texts governing inclusive methods, marked by breaks in discursive coherence, combine and interconnect in an apocryphal discourse underlying the way teachers and administrative personnel elaborate elements of practical epistemology. This subliminal and hybrid professional discourse, one of the factors of which is denial, can strengthen integrative practices, hinder the transformation of thèse practices and the use of innovative pedagogical forms by diverting inclusive methods from their purpose. This discourse allows contradictory elements or objects to coexist within it while refraining from dealing with these contradictions. As a result, everything functions as if the contradictions did not exist.

Keywords: Denial - Discordances - Discourse - Distortion - Hybridization - Inclusion - Innovation - Integration Intertextuality - Method - Pedagogical accessibility - Practical epistemology - Resistance. 

ANS une communication effectuée au IVe colloque Ophris (Observatoire des pratiques sur le handicap: recherche et intervention scolaire) de mars 2012 à Nancy, tout récemment publiée dans un ouvrage aux Presses universitaires de Nancy ${ }^{1}$, je me suis appuyé sur le concept d'obstacle épistémologique, tel que défini par Gaston Bachelard dans La Formation de l'esprit scientifique, pour analyser un corpus de discours tenus par des professionnels et des chercheurs au regard des objets de savoir relatifs au handicap et aux besoins éducatifs particuliers. La question était de savoir si nous disposons d'analyseurs efficaces au regard des résistances au paradigme de l'inclusion (Ebersold, 2009) qui s'incarnent dans le réseau complexe des discours professionnels, théoriques et institutionnels intriqués. La première piste théorique que l'on s'est proposé de suivre est celle de la "psychanalyse de la connaissance objective ", que Gaston Bachelard entreprend en 1938 (p. 3) en la déclinant notamment en "psychanalyse de l'erreur initiale " (p. 18) et en posant " en termes d'obstacles [...] le problème de la connaissance scientifique " (p. 13). Notre étude a fait apparaître que deux types d'obstacles, l'obstacle "verbal " (Bachelard, chap. 4 p. 73) et l'obstacle "substantialiste " (ibidem, chap. 6, p. 97), semblent permettre à la fois de rendre compte du melting pot terminologique et conceptuel qui caractérise ces discours et d'ouvrir des pistes de compréhension de quelques-uns des détournements et des distorsions notionnels qu'ils recèlent. II s'agissait d'une première étape dans l'élaboration d'une cartographie des impasses de l'inclusion, à travers les analyseurs psychanalytiques de la connaissance. Mais si I'on peut espérer ainsi approcher ce que l'on pourrait appeler, dans l'esprit de Gaston Bachelard, le soubassement de la connaissance objective et, en l'occurrence, celui du pensé et de l'agi éducatif et pédagogique au regard du paradigme de l'inclusion, la réflexion ne s'en trouve pas moins encadrée par une "psychanalyse de la raison" (Bachelard, p. 19) essentiellement fondée sur l'opposition entre l'observation et l'expérimentation ou, autrement dit, entre l'empirisme immédiat et la pensée scientifique et rationnelle. Une telle approche est profondément ancrée dans une épistémologie du sujet en tant qu'être de raison, comme en témoigne la dimension ontogénétique développée par Bachelard à travers ce qu'il appelle la " psychanalyse de l'erreur initiale » (p. 18):

Ils [les professeurs] n'ont pas réfléchi au fait que l'adolescent arrive dans la classe de Physique avec des connaissances empiriques déjà constituées: il s'agit alors, non pas d'acquérir une culture expérimentale, mais bien de changer de culture expérimentale, de renverser les obstacles déjà amoncelés par la vie quotidienne.

C'est pourquoi l'objet de cet article est tout d'abord d'élargir le cadre théorique de l'analyse à la dimension du discours (Foucault, 1971) en s'intéressant au fonctionnement et à la formation d'un territoire d'énoncés de type prescriptif, de façon à mettre en évidence certaines discordances intratextuelles et intertextuelles. On fait ensuite I'hypothèse que ces contradictions, dont la figure du déni est l'un des générateurs,

\footnotetext{
1. H. Benoit, "Les impasses de l'inclusion: obstacles théoriques et résistances langagières ", in J.-M. Perez, T. Assude, dir., Pratiques inclusives et savoirs scolaires: paradoxes, contradictions et perspectives, Presses universitaires de Nancy, Nancy, 2013.
} 
font écho, voire servent de référence, au processus d'élaboration par les acteurs de l'inclusion des éléments de leur "épistémologie pratique " (Sensevy, 2007).

\section{CADRE THÉORIQUE DE L'ANALYSE DES DISCOURS}

Les travaux de Michel Foucault permettent en effet de poursuivre la recherche des impasses du pédagogique face au paradigme de l'inclusion en s'affranchissant du cadre de l'épistémologie du sujet pour prendre en compte la dimension du discours, en tant que producteur d'objets de savoir et vecteur de pouvoir. Parce qu'il entreprend dans Les mots et les choses d'analyser les réseaux anonymes sous-jacents à I'histoire des discours, Michel Foucault offre la possibilité d'articuler aux analyseurs psychanalytiques de la connaissance, que sont les obstacles épistémologiques bachelardiens, des analyseurs de la formation des discours dans le domaine de l'inclusion scolaire et particulièrement dans celui des textes prescriptifs et explicatifs (loi, décrets et circulaires) qui se donnent explicitement pour but de bâtir les dispositifs inclusifs de L'école pour tous - formule officiellement labellisée par le ministère de l'Éducation nationale à travers le site web " ecolepourtous ${ }^{2}$ ". De la Leçon inaugurale au Collège de France prononcée le 2 décembre 1970, L'ordre du discours, publiée en 1971, se dégage un programme d'analyse du discours qui s'attache autant à la "part critique ", qui cherche à établir les principes de fonctionnement d'un territoire d'énoncés en élucidant les relations qu'ils entretiennent entre eux, qu'à la "part généalogique ", qui s'attache " aux séries de la formation effective du discours " et s'efforce d'identifier "son pouvoir d'affirmation ":

La part critique de l'analyse s'attache aux systèmes d'enveloppement du discours; elle essaie de cerner, de repérer ces principes d'ordonnancement, d'exclusion, de rareté du discours. [...] La part généalogique de l'analyse s'attache en revanche aux séries de la formation effective du discours: elle essaie de le saisir dans son pouvoir d'affirmation, [...] le pouvoir de constituer des domaines d'objets, à propos desquels on pourra affirmer ou nier des propositions vraies ou fausses. (p. 71-72)

C'est dans une perspective poststructuraliste que l'on s'attachera ici à analyser en quoi les ruptures de cohérence dans un microsystème discursif de textes prescriptifs concernant l'inclusion se combinent et s'articulent dans un discours apocryphe, susceptible de faire obstacle à la transformation des pratiques intégratives et d'entraver la mise en place de formes pédagogiques innovantes en détournant les dispositifs inclusifs de leur finalité déclarée. L'hypothèse est que ce discours de nature subliminale ou souterraine fait écho, voire sert de référence, au processus d'élaboration par les acteurs des éléments de leur "épistémologie personnelle " (Brousseau, 1986), de leur "idéologie épistémologique " (Perrin-Glorian, 1994) ou de leur "épistémologie pratique " (Sensevy, 2007). C'est à Sensevy que l'on doit l'expression d' "épistémologie pratique du professeur »: I'épistémologie pratique peut être définie comme une théorie sous-jacente à l'activité de l'enseignant qui naît de la pratique et qui la détermine sur la base de certaines théories qu'il s'est approprié au cours de sa formation et de son expérience (Théry, 2012). II s'agit d'un

2. <http://www.ecolepourtous.education.fr/accueil-scolarisationdes/v.html> 
système de références implicites relatif à la connaissance qui oriente les processus et les pratiques d'enseignement:

"Lorsqu'un professeur organise l'enseignement, il le fait notamment en fonction d'un certain nombre d'idées, plus ou moins explicites, qu'il entretient à propos du savoir lui-même, de la nature foncière de l'apprentissage, de la signification de l'enseignement " (Sensevy, 2007, p. 33)

L'épistémologie pratique fonctionne " comme un tropisme d'action qui surdétermine dans une certaine mesure le pilotage de la classe " (ibidem, 2006, p. 219) ; au-delà des intentions exprimées ou manifestées " il existe des déterminants généraux de l'activité du professeur, qui bornent son action par des ensembles de contraintes et de ressources [...] ". Nous nous posons la question de savoir dans quelle mesure les déterminants généraux de l'activité des enseignants peuvent être infléchis par les défauts de cohérence discursive de textes prescriptifs et constituer ainsi un obstacle à l'évolution des pratiques vers l'accessibilité pédagogique, en contribuant à la distorsion et au détournement des dispositifs inclusifs.

Le noyau du microsystème de textes prescriptifs que nous nous donnons comme corpus d'analyse est constitué par les textes réglementaires relatifs aux quatre principaux dispositifs inclusifs officiellement instaurés par les textes d'application de la loi du 11 février 2005 et qui, de fait, formalisent le contexte de travail et les missions de la plus grande partie des enseignants spécialisés (Capa-SH ${ }^{3}$ ) et $2 \mathrm{CA}-\mathrm{SH}^{4}$ ) dans les premier et second degrés: la Clis (Classe pour l'inclusion scolaire - 2009), I'Ulis (Unité localisée pour l'inclusion scolaire - 2010), I'UE (Unité d'enseignement - 2009) et le Pass (Pôle pour l'accompagnement à la scolarisation des jeunes sourds - 2010). Le choix de ces textes repose sur des critères précis qui sont au nombre de trois: 1) les dispositifs présentés constituent l'ensemble des déclinaisons du cadre générique du "dispositif adapté ", mentionné à l'article 1 du décret du 30 décembre $2005{ }^{5}$, dont le besoin indiqué dans le PPS justifie que l'élève handicapé fréquente un autre établissement scolaire que son établissement " de référence "; 2) le bénéfice de ces dispositifs est expressément subordonné à une décision de la $\mathrm{CDAPH}^{6}$, ce qui les distingue des nombreux autres dispositifs de droit commun (dispositifs d'aide à l'école élémentaire, aide personnalisée ou aide spécialisée avec le Rased 7 ; dispositifs d'aide et de soutien existant au collège; dispositifs relais...) qui n'exigent pas une telle décision; 3) ces dispositifs sont tous explicitement référés à l'inclusion scolaire (Clis et Ulis) ou à l'école inclusive (Pass), sauf I'UE qui est quant à elle référée à la scolarisation, sachant que le décret correspondant ${ }^{8}$ indique que dans I'article D 312-55 $2^{e}$ alinéa du Code de la santé publique "les mots: "à l'intégration scolaire"

3. Certificat d'aptitude professionnelle pour les aides spécialisées, les enseignements adaptés et la scolarisation des élèves en situation de handicap.

4. Certificat complémentaire pour les enseignements adaptés et la scolarisation des élèves en situation de handicap.

5. Décret 2005-1752 "relatif au parcours de formation des élèves présentant un handicap ".

6. Commission des droits et de l'autonomie des personnes handicapées, instaurée par la loi 2005-102 du 11 février 2005.

7. Réseaux d'aides spécialisées aux élèves en difficulté, circulaire 2009-088 du 17 juillet 2009.

8. Décret 2009-378 du 2 avril 2009 " relatif à la scolarisation des enfants, des adolescents et des jeunes adultes handicapés et à la coopération entre les établissements mentionnés à l'article L. 351-1 du code de l'éducation et les établissements et services médico-sociaux mentionnés aux $2^{\circ}$ et $3^{\circ}$ de l'article L. 312-1 du code de l'action sociale et des familles". 
sont remplacés par les mots: "à la scolarisation"». Le fait que les textes concernant les Egpa ne figurent pas dans le corpus, bien que leur rôle dans le contexte de l'ASH soit indéniable, tient au fait qu'ils sont clairement présentés comme des "structures

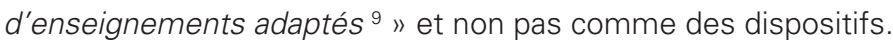

\section{DISCORDANCES DISCURSIVES INTERNES AUX TEXTES RELATIFS AUX CLIS}

\section{Du dispositif d'intégration...}

Dès 2002, la circulaire 2002-113 présentait la Clis comme un dispositif collectif (III - 1):

"Une intégration dans un dispositif collectif, la classe d'intégration scolaire, sera

proposée dès lors que les besoins de l'élève sont tels que des aménagements

substantiels doivent être apportés [...]"

et comme un dispositif d'intégration: "La Clis constitue dans l'école un dispositif d'intégration » (III- 3).

L'hétérogénéité des deux notions de classe et de dispositif (il ne viendrait à l'idée de personne de dire que tel élève est scolarisé dans un dispositif $6^{\bullet}$ ou un dispositif Terminale: on dit qu'il est en $6^{e}$; qu'il est en Terminale) était assumée par le texte qui s'appuyait sur l'idée de " classe ouverte " pour articuler l'une avec l'autre. C'est en tant que la Clis était une classe ouverte qu'elle pouvait être en même temps une classe et un dispositif d'intégration, c'est-à-dire un groupe-classe noyau à partir duquel devaient être organisés pour chaque élève scolarisé " des temps d'intégration dans des classes ordinaires, autant que ses moyens le lui permettent " (III - 3).

À cet égard, on relèvera l'ambiguïté du terme intégration dans le sigle Clis : celle-ci est-elle une classe d'intégration en tant que dispositif où l'on intègre un élève, ou parce qu'elle est une plate-forme de départ pour des intégrations en classe ordinaire au sein de l'école. La première intégration (dite collective) dans un groupe-classe réservé à des élèves handicapés et identifié en référence à un trouble - "Clis 1 : [...] enfants présentant des troubles importants des fonctions cognitives: retard mental global, difficultés cognitives électives, troubles psychiques graves, troubles graves du développement... " (III.2) - apparaissant comme le contraire de la seconde (dite individuelle), qui consiste précisément à défaire la première. Cette situation paradoxale résultait de la coexistence, au sein du système éducatif, de deux filières, I'une ordinaire, l'autre spécialisée, qui conduisait à fonder le fonctionnement intégratif sur le passage pour les élèves concernés de l'une à l'autre voie (Benoit, 2012a, p. 68).

\section{au dispositif inclusif}

En juillet 2009, lorsque paraît la circulaire instaurant les Classes pour l'inclusion scolaire (Clis) en lieu et place des Classes d'intégration scolaire (Clis), la loi de 2005 a mis en place un nouveau paradigme, celui de la scolarisation/inclusion en remplacement de celui de l'intégration. La nouvelle philosophie inclusive peut se résumer en deux points:

9. Circulaire 2006-139 du 29 août 2006 «Enseignements généraux et professionnels adaptés dans le second degré (Egpa) ». 
- L'établissement de référence pour la scolarisation de tout élève est toujours l'établissement ordinaire le plus proche de son domicile (décret du 30 décembre 2005) ;

- Les "dispositifs adaptés " évoqués à l'article 1 du même décret du 30 décembre 2005 "relatif au parcours de formation des élèves présentant un handicap " ont vocation à accompagner et à faciliter cette scolarisation et non pas à prendre la forme de classes spécifiques constituant une filière parallèle, à partir de laquelle il faudrait redoubler le processus d'intégration: après avoir intégré d'abord l'élève concerné dans la classe/dispositif, on déclencherait secondairement des intégrations dans une ou plusieurs classes ordinaires.

Le titre 1 du nouveau texte du 17 juillet 2009 sur les classes pour l'inclusion scolaire reprend cette idée en définissant la Clis comme: "un dispositif de scolarisation installé dans une école élémentaire ou maternelle ". C'est également de la même manière qu'est explicité par l'arrêté du 2 avril 2009 le rôle du dispositif pédagogique de l'Unité d'enseignement (UE) qui est de s'appuyer " sur les enseignements que ces élèves reçoivent dans leur établissement scolaire de référence ou dans l'établissement scolaire dans lequel ils sont scolarisés " afin de permettre à chaque élève "de réaliser, en référence aux programmes scolaires en vigueur, en complément ou en préparation de l'enseignement reçu au sein des établissements scolaires, les objectifs d'apprentissage " (article 2a). II s'agit bien là de la description d'un dispositif à repérage externe (Benoit, 2012a, p. 69), ou hétéroréférentiel, dont la visée est centrée sur l'inclusion des élèves concernés dans des classes et établissements scolaires ordinaires et non plus sur un projet pédagogique intrinsèque, comme c'est le cas d'une classe constituée comme telle dont le fonctionnement est institutionnellement autoréférentiel (projet de classe, maître de la classe, etc.).

C'est néanmoins une approche très différente, celle de la classe, qui est développée à la fin du titre 1 et au titre 2 de ce même texte sur les classes pour l'inclusion scolaire:

- La Clis est une classe placée sous la "responsabilité " d'un maître: "I'enseignant de la classe".

- "La Clis est une classe à part entière de l'école..."

- "La Clis est prise en compte, au même titre que les autres classes de l'école..." À partir de cette classe, les élèves pourront "fréquenter autant que possible une classe ordinaire " (titre 4), c'est-à-dire passer d'une classe spécifique à une classe ordinaire pour une durée limitée, ce qui correspond très exactement au paradigme intégratif, dans le cadre des échanges entre les filières caractéristiques du dualisme éducatif (Benoit, 2003, p. 82). Le texte précise que les enseignants de l'école doivent s'attendre à recevoir des élèves en intégration, qui restent donc étrangers à leur classe. Où est la plus-value de l'inclusion, qui est pourtant affichée dans l'intitulé même du dispositif? Et en quoi s'agit-il d'un dispositif?

\section{Éléments induits en termes d'épistémologie pratique}

1) Les termes inclusion et intégration renvoient aux mêmes pratiques consistant à organiser dans un groupe classe d'élèves handicapés des temps limités de scolarisation dans une classe ordinaire. 
L'équivalence des termes inclusion et intégration se trouve implicitement établie pour les enseignants comme pour tous les autres acteurs du système éducatif. L'évolution du lexique professionnel peut être par conséquent indexé à un effet de mode, voire à une harmonisation et à une euphémisation politiquement correctes, liées à la mondialisation des fonctionnements éducatifs.

2) Les temps passés par un élève handicapé en dehors de sa Clis sont appelés des temps d'inclusion (et non plus des temps d'intégration) et l'élève concerné sera désigné dans les échanges entre enseignants comme un élève en inclusion (et non plus élève en intégration).

Or les notions d'intégration et d'inclusion ne se, situent pas au même niveau et l'expression temps d'inclusion tend à gommer ce décalage conceptuel. L'intégration renvoie à un ensemble de pratiques circonscrites dans un hic et nunc professionnel, tandis que la scolarisation en milieu ordinaire ne représente pas au titre de l'inclusion " une fin en soi " (Armstrong F., Armstrong D., Barton L., 2000) et ne prend tout son sens qu'à partir du moment où elle embrasse à la fois toutes les phases du parcours de vie et tous les systèmes sociaux et culturels qui pourront avoir sur lui un impact direct ou indirect.

3) Le projet de clis est premier: il fédère les activités et donne son identité et son dynamisme au groupe d'élèves; les temps d'inclusion sont programmés en second lieu.

L'intitulé du dispositif ( " classe pour l'inclusion scolaire ») présente l'inclusion comme objectif premier et fondateur, mais la visée du fonctionnement de la Clis reste très ambiguë: d'un côté "Le projet de la Clis est élaboré et mis en œuvre par l'enseignant qui y est affecté " et "tous les élèves de la Clis reçoivent un enseignement adapté de la part de l'enseignant de la Clis ", ce qui laisse entendre que l'organisation pédagogique autoréférencée du groupe classe est prioritaire; de l'autre, ce projet de Clis doit être conçu "en articulant entre eux les objectifs visés par les projets personnalisés de scolarisation des élèves concernés " dont la mise en œuvre peut les conduire "à fréquenter, autant que possible une classe ordinaire ". II y a là une logique du " même si " qui renverse l'ordre des injonctions et peut produire des situations de dilemme professionnel susceptibles de mettre en difficulté face à l'inspection pédagogique un enseignant qui privilégierait l'inclusion par rapport au projet de classe.

4) Les inclusions devront être préparées en fonction des capacités d'adaptation des élèves concernés, donc de leur intégrabilité. II faut donc établir des critères (comportementaux ou scolaires: pouvoir parler, savoir écrire...) et tenir compte du niveau de tolérance de l'enseignant ordinaire.

Le discours idéologique de la capacité, parfois désigné sous le nom d'ableisme par transposition du terme anglais ableism, a été identifié et analysé par les chercheurs dans le domaine des Activités physiques adaptées (APA). Pour Lisette Burrows, la vision poststructuraliste soulève la question de l'origine et de la finalité des normes: " elle fait aussi réfléchir sur notre propension à maintenir des hiérarchies fondées sur 
les capacités dans le domaine de l'éducation et dans la vie en général " (Burrows, 2012, p. 14). La centration sur les capacités est symétrique d'une approche par les incapacités, qui conduit à une focalisation sur les dysfonctionnements personnels de l'élève plutôt que sur l'accessibilisation de la situation d'apprentissage (Benoit, 2008, p. 100). Tandis que l'intégrabilité, la scolarisabilité sont mesurées l'une et l'autre à l'aune des capacités personnelles et reposent donc sur les épaules de l'élève concerné et de son entourage (Plaisance, 2009), I'accessibilité est au contraire de la responsabilité des acteurs du système éducatif dont le rôle est de transformer les cadres et les modalités d'enseignement de façon qu'ils puissent répondre de manière adaptée aux besoins de la diversité des apprenants. Car l'éducation inclusive ne vise pas à satisfaire les besoins d'une minorité vulnérable dans des situations ordinaires mais se réalise par la transformation en profondeur du fonctionnement des écoles ordinaires pour les rendre accessibles, de manière permanente et non pas seulement temporaire, à tous les élèves (D'Alessio 2008, p. 45; Ainscow, 1997, p. 5).

5) Les enseignants ordinaires de l'école sont légalement tenus d'accepter de scolariser un élève handicapé, mais il vaut mieux négocier les temps d'inclusion pour éviter les conflits.

On assiste ici à la construction d'un positionnement professionnel du maître responsable de Clis que l'on peut caractériser par un "modèle " ou une "figure " (Jeanne, 2007, p. 92), qui se trouve au fondement de la logique de l'action, celle de l'ambassadeur du handicap, cherchant à faire valoir les droits de ses ressortissants au regard des autorités administratives du pays étranger dans lequel ils souhaitent séjourner temporairement. Le statut d'élève en intégration au sein d'un système éducatif peut en effet être décrit en terme de précarité scolaire (Benoit, 2012a, p. 70), en ce qu'il présente dans son fonctionnement une double isomorphie ${ }^{10}$ à celui du travailleur précaire (qui renvoie à la notion de contrat à durée déterminée) et à celui de personne étrangère à un territoire national, placée sous la menace d'une reconduite à la frontière (en l'occurrence celle du milieu scolaire ordinaire), si elle ne fait pas preuve des capacités d'adaptation requises (par exemple, savoir correctement s'exprimer dans la langue du pays d'accueil).

6) Les temps d'inclusion peuvent constituer un danger de souffrance pour l'élève fragile tandis que la Clis est un cocon rassurant et propice au développement dans le respect de la différence.

À la figure de l'ambassadeur, qui apparaît quand il s'agit des relations avec les autres enseignants de l'établissement, se combine chez le maître de la Clis celle de la mère protectrice, au regard de l'encadrement des élèves qui lui sont confiés. Ce désir de protection " est soutenu par des attitudes maternantes que pourraient traduire les mots utilisés pour désigner les élèves: "mes petits," "mon gamin," "mon petit

10. Le terme d'isomorphisme (Benoit, 2005, p. 50; Curchod-Ruedi \& Doudin, 2013, p. 236) est à I'origine un concept mathématique qui signifie que chaque élément d'un ensemble correspond à un élément d'une autre ensemble, chacun de ces éléments jouant respectivement le même rôle dans son ensemble d'origine. La transposition de cette notion aux systèmes linguistiques ou humains met l'accent sur la correspondance des relations à l'intérieur des différents systèmes. 
bouchon." À ce titre, la salle de classe spécialisée " est conçue comme un espace sûr et douillet, un lieu d'apaisement. " (Ployé, 2013, à paraître).

\section{DISCORDANCES DISCURSIVES INTERTEXTUELLES: CLIS, UPI, ULIS...}

\section{Évolution imbriquée des Clis et des Ulis}

En 1995, puis en 2001, les textes définissant les UPI (Unités pédagogiques d'intégration) introduisent une nouvelle notion, celle de dispositif collectif de scolarisation:

" I/ convient d'être particulièrement attentif à ce que ces unités ne constituent pas une "filière," mais bien un dispositif ouvert sur l'établissement scolaire, même lorsqu'il s'avère opportun de prévoir, pour certaines activités le regroupement des élèves concernés " (circulaire 2001-035 du 21 février 2001)

Ce dispositif n'est jamais désigné sous le nom de classe: le principe du regroupement impliquant que les classes de référence des élèves concernés sont les classes ordinaires. L'intention du texte est clairement de créer dans le second degré un dispositif hétéroréférentiel qui ne soit pas comparable à une classe constituée, telle que la Clis (classe d'intégration scolaire) a été définie par la circulaire princeps du 18 novembre 1991.

C'est dans un texte paru dix ans plus tôt, le 9 avril 1990, dont l'objet était de créer les Rased (Réseaux d'aides spécialisées aux élèves en difficulté) qu'apparaît pour la première fois la modalité de fonctionnement scolaire consistant à regrouper temporairement des élèves pour des actions de soutien, sans que ceux-ci ne cessent d'être inscrits dans leur classe d'origine:

"Les aides spécialisées à dominante "pédagogique" peuvent être organisées

[... par l'organisation de regroupements d'adaptation rassemblant de manière temporaire des élèves en difficulté qui continuent à fréquenter la classe ordinaire dans laquelle ils demeurent régulièrement inscrits. " (circulaire 90-082 du 9 avril 1990, 2.1., abrogée remplacée par la circulaire n²002-113 du 30 avril 2002).

Cette modalité du regroupement d'adaptation sera par la suite transposée dans le domaine de la scolarisation des élèves handicapés pour fonder les UPI (en 1995, puis en 2001), ensuite les Ulis (en 2010), et confirmée pour les Rased en 2002 (circulaire $n^{\circ} 2002-113,11.4$ ).

En 2002, un souci d'harmonisation conduit le ministère de l'Éducation nationale à redéfinir le fonctionnement des Clis dans le cadre de la partie III de la circulaire 2002-113 du 30 avril 2002, que nous avons analysée plus haut. Sous I'influence de I'UPI, La Clis connaît ainsi une hybridation, elle devient un objet composite, mi-classe, mi-dispositif, une "classe ouverte ", qui fait écho au "dispositif ouvert " que constitue I'UPI.

En 2010, la création des Ulis (Unités localisées pour l'inclusion scolaire), qui se substituent aux UPI, apparaît comme l'aboutissement de la logique du dispositif hétéroréférencé, puisque le texte de la circulaire 2010-088 du 18 juin 2010 indique très clairement que les élèves concernés sont inscrits dans les divisions du collège (c'est-à-dire en $6^{e}$, en $4^{e}$, etc.) et bénéficient de l'aide nécessaire à leur scolarisation par le biais du dispositif. 
Mais cette évolution remarquable des conditions de scolarisation des élèves handicapés dans le second degré a été précédée, à peine un an plus tôt, par la circulaire du 17 juillet 2009 sur les Clis, dont on a vu qu'elle fait retour au paradigme intégratif et vide ainsi de sa cohérence le principe du dispositif en l'assignant à un fonctionnement de filière autoréférencée dans lequel la classe spécifique est le point de départ obligé des "inclusions individuelles".

L'hésitation des discours prescriptifs, qui se présente comme une forme d'hybridation intertextuelle, comparable à l'hybridation intratextuelle, est à la source d'un discours apocryphe et souterrain (au sens où les juristes commencent à travailler sur le concept de "droit souterrain ") qui est susceptible de nourrir l'épistémologie pratique des enseignants et des personnels d'encadrement (qui sont pratiquement tous d'anciens enseignants). Cette discordance intertextuelle s'observe de surcroît entre des textes relatifs au second degré (inclusifs) et des textes relatifs au premier degré (traditionnellement intégratifs), alors même que c'est majoritairement à des enseignants spécialisés du premier degré, plus nombreux, auxquels on demandera, au titre de coordonnateur d'UPI, puis d'Ulis de mettre en œuvre les dispositifs inclusifs innovants du second.

\section{Éléments induits en termes d'épistémologie pratique}

1) L'Ulis, comme I'UPI, fonctionne approximativement comme une Clis de collège : des temps d'inclusion sont organisés dans les classes ordinaires en fonction des possibilités des élèves.

Parce que les principaux des collèges ont été amenés à affecter, au moment de sa création, un local au dispositif UPI ou Ulis, un glissement sémantique s'est produit de la notion de classe (en tant que salle) à la notion de classe (en tant que groupe d'élève constitué). Ainsi le visiteur est-il souvent convié à visiter la classe UPI ou la classe Ulis. II est à cet égard significatif que le terme "ulyssiens " se soit spontanément développé pour désigner les élèves bénéficiant de ce dispositif. Si valorisant culturellement puisse-t-elle paraître au premier abord, cette appellation n'en renvoie pas moins à l'errance maritime d'un groupe d'hommes en quête de leur terre d'origine et abordant sans cesse des contrées étrangères comme autant d'étapes temporaires au cours de leur interminable voyage.

2) Le niveau des apprentissages au collège est souvent en grand décalage avec les compétences acquises par les élèves handicapés de l'Ulis. Les disciplines académiques ne sont pas les plus propices à l'inclusion et il vaut mieux préférer I’EPS, les arts plastiques et la musique.

Les notions d'intégration et d'inclusion ne se situant pas, comme on l'a dit, au même niveau conceptuel, la première peut être sélective dans le temps, l'espace et l'esprit, puisqu'elle se satisfait, en tant que modalité pratique d'éducation, de sa mise en œuvre concrète dans un cadre spatio-temporel et disciplinaire déterminé. Parce que son enjeu est le devenir de l'élève et non pas seulement l'élève lui-même, la seconde s'attache à identifier et à éliminer les barrières et les ruptures de tous ordres susceptibles de fragmenter l'unité du projet de formation: 
"Placer le devenir de la personne au centre incite à inclure dans la définition et le suivi du projet les facteurs source de ruptures durant la scolarisation des élèves à BEP et les moyens, si possible, de les prévenir, ces facteurs étant partie prenante du processus de production du handicap tant ils vulnérabilisent les individus. " (Ebersold, 2012, p. 61)

3) L'enseignant responsable de I'Ulis est un spécialiste du handicap, il connaît bien les troubles des élèves de son groupe et son expertise est indispensable aux professeurs disciplinaires qui ne peuvent rien sans lui.

Considérer que " ce n'est pas en soi le diagnostic qui détermine le choix de l'aide mais la nature des besoins de l'élève ${ }^{11}$ " conduirait cependant à mettre à distance la recherche étiologique du trouble, c'est-à-dire de la cause médicale des difficultés qui sont observées. C'est en tant qu'il serait déterminant pour l'action pédagogique et éducative que le diagnostic est à relativiser: une telle démarche met en avant, de manière pragmatique, l'observation de l'enfant en situation d'apprentissage par l'enseignant de la classe et non pas seulement par l'enseignant spécialisé ou le professionnel de santé. On n'est plus dans une logique de savoir dogmatique où tel type d'atteinte entraînerait inévitablement tels types de répercussions; on adopte une méthode dans laquelle on commence par observer, pour ensuite réfléchir aux modalités d'aide. Modalités d'aide qui consistent en fait à identifier les obstacles auxquels l'élève se heurte dans son environnement scolaire. On peut dire que tel élève a des difficultés pour comprendre les consignes, mais on peut aussi exprimer les choses différemment: on peut dire que cet élève ne comprendra la consigne qu'à la condition qu'on la lui explique de manière claire et qu'on la lui répète un certain nombre de fois. On peut dire que tel élève à des difficultés de repérage dans l'espace, mais on peut dire aussi que cet élève a besoin d'un cadre structuré pour se repérer dans l'espace. Le fait que les modalités de l'aide et les adaptations pédagogiques ne relèvent pas d'une prescription spécialisée d'ordre médical, mais de l'observation de l'enseignant constitue un élément fort de son identité professionnelle dans le nouveau contexte inclusif de scolarisation des élèves en situation de handicap. Dans cette perspective, les difficultés d'apprentissage ou d'adaptation scolaire ne sont pas à rapporter à de supposés dysfonctionnements psychiques intrinsèques, mais à analyser dans le cadre de l'activité de l'apprenant en situation. Mais la prégnance de l'approche médicale peut conduire à majorer le poids des pratiques de diagnostic a priori, fondées sur l'identification de besoins éducatifs a priori, construits comme l'envers positif de la répercussion du trouble (Benoit, 2012a, p. 76).

4) les inclusions individuelles dans les classes ordinaires doivent rester strictement sous contrôle et nécessairement limitées au risque de devenir sauvages et de faire souffrir ensemble professeurs et élèves handicapés.

Pour Alexandre Ployé, ce type de collaboration entre les enseignants du collège et le coordonnateur de I'Ulis " aboutit néanmoins à une certaine restriction,

11. Référentiel de compétences pour les formations aux Capa-SH et 2CA-SH donné par la circulaire du 10 février 2004, annexe 11 . 
quantitative et qualitative, des projets d'inclusion [...] chacun peut préserver son identité; le partage des cultures demeure limité et le choc de la transformation du collège par le projet inclusif s'en trouve atténué " (Ployé, 2013, à paraître). Cette restriction s'explique par le fait que l'acteur spécialisé se représente son domaine de compétence comme un territoire qui s'arrêterait là où commence celui de ses collègues du milieu ordinaire. Pour la surmonter, il lui faut intégrer l'idée que ces deux domaines se recouvrent et se superposent. Dès lors, ses fonctions ne relèvent plus du registre de la substitution, mais de celui de la combinaison et de l'articulation des compétences dans la dynamique d'un réseau de ressources: il s'agit donc pour lui de passer d'une grammaire professionnelle de type paradigmatique, dans laquelle les déclinaisons de chaque type d'interventions professionnelles se déroulaient parallèlement, à une grammaire de type syntagmatique, où les rôles interagissent en permanence dans un environnement continu, structuré par ses objectifs et non plus par ses frontières.

5) En lycée particulièrement, des critères de niveau scolaire doivent être fixés sur la base des référentiels de compétences du CAP. Les élèves ne satisfaisant pas à ces critères relèvent des IMPro.

Réintroduire le principe d'une sélection sur la base de la maîtrise de compétences scolaires à l'entrée d'un dispositif visant l'adaptation des enseignements de l'enseignement secondaire consiste à revenir à des pratiques intégratives, fondées sur la capacité de l'élève à répondre aux exigences de l'école, tandis que la perspective inclusive conduit à s'engager dans la mise en accessibilité des situations d'apprentissage scolaire. La circulaire du 18 juin 2010 sur la mise en place des Ulis se trouve à ce titre en discordance intertextuelle avec les textes de 2009 concernant le " socle commun de connaissances et de compétences ${ }^{12}$ ". La réponse au problème de l'écart de performances par la sélection des élèves et par la filiarisation consiste à orienter très précocement les élèves et à séparer les plus performants - destinés à suivre des cursus d'excellence - et les plus faibles - destinés à des parcours spécialisés - de la masse des élèves. L'expérience ${ }^{13}$ a montré, en France et en Allemagne notamment, selon des modalités différentes, que cette formule a tendance à creuser les écarts plutôt qu'à les réduire, mais qu'elle permet de les gérer. Les pratiques d'intégration scolaire sont parties prenantes de ce mode d'organisation, au sens où elles font reposer le maintien en milieu scolaire ordinaire de l'élève handicapé sur les capacités d'adaptation scolaire dont il va se montrer capable (Benoit, 2012b, p. 118). La logique est celle de la confrontation à la norme et de la mise à l'épreuve, faute de quoi, la réorientation en milieu spécialisé s'impose.

12. $B O \mathrm{n}^{\circ} 40$ du 29 octobre 2009.

13. Les résultats de l'enquête PISA 2009, la première d'un nouveau cycle triennal, et dont les derniers résultats ont été publiés le 7 décembre 2010, mettent en évidence un écart de performances scolaires, qui s'est accentué de plus en plus au cours des dix dernières années, au point d'apparaître aujourd'hui comme une véritable dichotomie, entre les élèves qui réussissent et ceux qui n'acquièrent pas les compétences attendues. 


\section{EN CONCLUSION: LA FIGURE DU DÉNI}

L'un des principes générateurs d'hybridation intra et intertextuelle des discours est la figure du déni. André Meynard (2010, p. 22) évoque, à propos du risque de mise à l'écart des langues signées, cette notion freudienne de Verleugnung, processus psychique par lequel on prend connaissance et l'on admet l'existence d'une réalité, tout en conservant intacte la croyance inverse qui l'invalide. Ainsi peut-on connaître sans reconnaitre, c'est-à-dire refuser de prendre connaissance et particulièrement de tirer les conséquences au niveau symbolique (Bon, 2004) d'une réalité que l'on perçoit ou d'un acte que I'on commet. On est proche de la notion juridique de non-lieu, qui peut correspondre à un déni de justice. Dans le domaine de la fiction cinématographique, I'expression utilisée par Octave Manonni (1969) " Je sais bien, mais quand même... " dans le titre d'un article où il analyse le spectateur comme un sujet clivé vis-à-vis du spectacle auquel il assiste, permet de se représenter le fonctionnement de la figure du déni comme générateur et organisateur de discours hybrides inhibiteurs d'innovation scolaire. De même que le spectateur est clivé en ce sens qu'il n'y croit pas mais quand même - et cela sans qu'il y ait antagonisme entre celui qui, en lui, y croit, et celui qui n'y croit pas, le discours et le dispositif articulent en eux-mêmes, sous le régime du même si $^{14}$, des éléments ou des objets contradictoires, en s'abstenant de prendre en charge ces contradictions, de sorte que tout se passe comme si elles n'existaient pas.

On pourrait par exemple illustrer autrement ce processus en pointant la concomitance, dans un même temps social, d'une loi de 2005 reconnaissant la langue des signes française comme une langue à part entière, assortie d'un droit à l'enseignement pour les "élèves concernés ", et de l'accord donné en 2007 par la Haute autorité de la santé (HAS, 2007, p. 32) à la généralisation d'un "dépistage néonatal de masse " dans les deux jours après la naissance, en vue de pouvoir recourir aux implants cochléaires avant un an pour traiter la maladie-surdité. II est clair qu'un tel dépistage médical signifie le rejet de l'appréhension sociale et anthropologique de la langue des signes, de laquelle découlent les perspectives d'éducation fondée sur la communication signée.

II est clair que les textes prescriptifs ne recouvrent pas la réalité sociale des pratiques qu'ils sont censés encadrer et que l'évolution des comportements professionnels dépend d'une pluralité de facteurs, parmi lesquels les conditions de la formation jouent un rôle déterminant. Mais I'opposition que l'on allègue souvent entre ces prescriptions et la réalité du terrain, dont les acteurs seraient imperméables aux nouvelles orientations, est sans doute trompeuse et mérite d'être repensée à la lumière de l'incidence des discordances des discours injonctifs en termes d'élaboration de l' "épistémologie pratique » et d'inhibition de l'innovation inclusive.

14. "Tous les élèves de la Clis reçoivent un enseignement adapté de la part de l'enseignant de la Clis, même si la mise en œuvre de leurs projets personnalisés de scolarisation conduit ces élèves à fréquenter, autant que possible une classe ordinaire. ", circulaire 2009-087 du 17 juillet 2009, 4. 


\section{Bibliographie}

AINSCOW (M.), "Towards inclusive schooling", British Journal of Special Education, 24, 1997, p. 3-6.

Armstrong (F.), Armstrong (D.), BARTON (L.), eds, Inclusive Education. Policy, Contexts and Comparative Perspectives, David Fulton Publisher, London, 2000.

BACHELARD (G.), La Formation de l'esprit scientifique, Bibliothèque des textes philosophiques, J. Vrin, Paris, 1986, $257 \mathrm{p}$.

BENOIT (H.), "Les impasses de l'inclusion: obstacles théoriques et résistances langagières " in J.-M. PEREZ, T. ASSUDE, dir., Pratiques inclusives et savoirs scolaires: paradoxes, contradictions et perspectives, Presses universitaires de Nancy, Nancy, 2013.

BENOIT (H.), "Pluralité des acteurs et pratiques inclusives. Les paradoxes de la collaboration ", La nouvelle revue de l'adaptation et de la scolarisation, $n^{\circ} 57$, 2012a, p. 66-78.

BENOIT (H.), "Comment répondre au défi de la diversité des élèves ", Une éducation pour la Suisse du futur - De la sélection à l'intégration, Académies suisses des sciences, Berne, 2012b, p. 117-122.

BENOIT (H.), " De la reproduction des pratiques à leur transformation: le défi de la formation des enseignants ", Reliance, 27, dossier "Faire culture commune ", Érès, 2008, p. 99-104.

BENOIT (H.), "Peut-on parler de besoins éducatifs particuliers en cas de difficulté scolaire? ", La nouvelle revue de I'AIS, n²2, 2003, p. 81-88.

BON (N.), La logique du fantasme, Intervention au séminaire de I'Association lacanienne internationale, Paris, 26-29 août 2004, <http://www.freud-lacan. com/articles/article.php?url_article=nbon100904\#notes $>$

BURROWS (L.), "De la théorie à la pratique: APA et poststructuralisme ", La nouvelle revue de l'adaptation et de la scolarisation, $n^{\circ}$ 58, 2012, p. 13-26.

CURCHOD-RUEDI (D.) \& DOUDIN (P.-A.), " Le soutien social aux enseignants: un facteur de réussite de la démarche inclusive ", La nouvelle revue de l'adaptation et de la scolarisation, $n^{\circ}$ 60, 2013, p. 229-244.

D'ALESSIO (S.), " 30 ans d'Integrazione scolastica en Italie. Réflexions critiques et suggestions sur le développement de I'éducation inclusive en Italie. ", La nouvelle revue de l'adaptation et de la scolarisation, in "L'éducation en France et dans le monde », H. BENOIT \& É. PLAISANCE, dir., Hors série n 5, 2008, p. 35-50.

EBERSOLD (S.), "Parcours de scolarisation et coopération: enjeux conceptuels et méthodologiques ", La nouvelle revue de l'adaptation et de la scolarisation, $\mathrm{n}^{\circ}$ 57, 2012, p. 55-64.

EBERSOLD (S.), "Autour du mot inclusion », Recherche et Formation, n 61, 2009. FouCAULT (M.), L'ordre du discours, Leçon inaugurale au Collège de France prononcée le 2 décembre 1970, Gallimard, 1971, 82 p.

FOUCAULT (M.), Les mots et les choses, Gallimard, Tel, réédition 2008, 1966, 400 p. HAUTE AUTORITÉ DE SANTÉ, SERVICE ÉVALUATION MÉDICO-ÉCONOMIQUE ET SANTÉ PUBLIQUE, Évaluation du dépistage néonatal systématique de la surdité permanente bilatérale, Paris, HAS, 2007. <http://www.has-sante.fr/portail/upload/docs/ application/pdf/rapport__evaluation_du_depistage_neonatal_systematique_ de_la_surdite_permanente_bilaterale.pdf> 
JEANNE (Y.), "Penser la violence en situation éducative: au fondement des logiques d'action ", La nouvelle revue de l'adaptation et de la scolarisation, $\mathrm{n}^{\circ} 38$, 2007, p. 91-99.

MANNONI (O.), Je sais bien, mais quand même..., 1963, Clefs pour l'imaginaire ou l'Autre Scène, coll. "Points ", Seuil, Paris, 1969.

MEYNARD (A.), "Quand une filière de soin fait taire l'enfance Sourde... ", La nouvelle revue de l'adaptation et de la scolarisation, n 49, 2010, p. 21-35.

PLAISANCE (É.), Autrement capables. École, emploi, société: pour l'inclusion des personnes handicapées, coll. "Mutations ", Autrement, Paris, 2009.

PLOYE (A.), " Collaborer à des démarches d'inclusion au collège: analyse clinique des modalités pédagogiques et des éprouvés psychiques des acteurs ", La nouvelle revue de l'adaptation et de la scolarisation, $n^{\circ}$ 61, 2013, à paraître.

SLEE (R.), "Limits to and possibilities for educational reform", International Journal of Inclusive Education, 10 (2-3), 2006, p. 109-119.

THÉRY (M.), ASSUDE (T.), dir., "Faire travailler ensemble tous les acteurs de l'inclusion?", dossier, La nouvelle revue de l'adaptation et de la scolarisation, $n^{\circ} 57,2012$, p. 7-206. 